\title{
Leap Motion Sensor for Natural User Interface
}

\author{
Nenad BRESLAUER, Irena GALIĆ, Mihael KUKEC, Ivan SAMARDŽIĆ
}

\begin{abstract}
In Human Computer Interaction $(\mathrm{HCl})$ research area, there is an increasing tendency to make devices as simple and as natural as possible for use. These devices are aiming to make input and output techniques, interaction, etc., easier. In the input domain, sensors monitor and interpret head, eye, face and even whole body movements. Interacting with the computer, hands are the most effective tool of general purpose, due to their functionality in communication and manipulation. Using hands as an input device is an attractive method for ensuring interaction between man and computer. This paper gives an overview of the Leap Motion devices as a technology that enables natural interaction between man and computer in NUI (natural user interface) implementation. The main idea to maximize the naturalness of the user environment and the use of hand movements is in the application of the sensor fusion of two Leap Motion devices. Applying sensor fusion of two Leap Motion devices will increase the range of hand movement and the interaction within the environment, which will again contribute to the natural user interface (NUI). The suggested method can be used offline or in real time, and can benefit from a wide range of applications, where the gesture of the hand and fingers is the focus of significance and estimation effect.
\end{abstract}

Keywords: gesture recognition technology; $\mathrm{HCl}$; Mid-air gestures; NUI

\section{INTRODUCTION}

The current operating systems are in the graphical user interface GUI where keyboard and mouse usage are practical for switching from applications and executing commands over them. The development of virtual and augmented reality as well as various $3 \mathrm{D}$ visualization and computer games have moved to 3D space. Visualization of these $3 \mathrm{D}$ worlds can be projected on screen or stereoscopic viewing devices. Controlling these $3 \mathrm{D}$ worlds with the keyboard and the mouse is not easy, or is even unnatural, according to some authors [15].

The human-computer interaction (HCI) is an important part of everyday life and is an important area of research that is gaining increasing attention with the emergence of new interaction devices such as Nintendo Wii Remote, Microsoft Kinect, Leap Motion etc. The aim of humancomputer interaction (HCI) is to make the interaction of man and computer as natural as possible, that is, to make it similar to human interaction [20]. In recent years, research of hand movement and gesture has become increasingly important in interaction between man and computer in the context of the user interface. The area of recognition and interpretation of hand gestures is a challenge for designers of a new kind of user interface based on natural human gestures. The direct use of hand gestures as a data entry interface is a very convenient and attractive way to achieve natural communication between man and computer [10, 24]. It strives to make the user interface almost invisible, inconspicuous and enables the user to master the computer, that is, to interact with the computer as quickly as possible. Such user interface is called NUI (Natural User Interface). NUI is not a natural user interface, but an interface that makes computer interaction more natural [25]. Users generally prefer multimodal interaction when available and intuitive to use [18, 23]. Particularly interesting are sensors for tracking hand movements and gestures. Movementtracking devices can be divided into two categories: (i) those that must be physically attached to the user or user has to hold them in their hands i.e. wearable devices, and (ii) those that follow movements and gestures without any physical contact, such as optical sensors.
Wearable devices for tracking hand movements and gestures can be performed in the form of gloves placed on the arm and with cables connected to the computer. Data glove is one of such sensing devices based for hand motion and gesture recognition using motion sensors such as accelerometer, gyroscope, bend sensor, force sensor, and so on. In paper: i) Tarchanidis et al. [21] developed a data glove based on force sensors that are attached to each finger. This data glove was used to detect the tactile sensation, but it had limitations of hand motion tracking and precise recognition. ii) Bui et al. [1] developed a data glove based on two-axis accelerometers positioned on the back of the Palm and each finger. However, hand gesture recognition using this device was only possible in two dimensions. These wearable devices have their own limitations.

The second category of movement tracking devices follows movements without physical contact, thus giving the user more freedom in interaction. This category of sensors allows more natural motion without gloves or wearable devices. The common term for such interaction found in literature enabled by tracking devices without physical contact with the user is "mid-air interaction" and "mid-air gestures" [12].

\section{BIMANUAL MID-AIR INTERACTION INTERFACE}

Mid-air interaction or in-air interaction and mid-air gestures or in-air gestures are terms found in the literature and recent research papers $[3,4,8,16]$ denoting interaction with computer system which user performs mainly using her or his upper extremities. Sensory device transforms movements of the one or more upper body extremities (arms, hands, palms, fingers) into data stream which is decoded and interpreted by computer algorithm as a "gesture". Gestures, i.e. hand and finger movements, which are performed by user accordingly to previously established pattern and recognised by algorithm as such can trigger action.

Sensory devices used in mid-air interaction systems, as described in the previous section of this paper, can be divided in two main groups: (i) wearable; those that are worn by user and (ii) those that the user has no physical 
contact with. The latter setup is also called "come as you are" $[22,24]$ as user does not wear any sensors, markers nor does the system need some special conditions. However, other than this classification it is rather amiss and impractical to differentiate methods and hardware used as there are many different approaches. What is more, every model researched unveils advantages and limitations of each category and strategies researched.

Better understanding of mid-air gestural interaction can be attained through a holistic approach to the topic. Putting aside technical challenges [13], there are many psychical [8], psychological [4] and psychophysical [6] aspects of using different approaches and sensors to achieve mid-air gestural interface. General "rule of thumb" of HCI and interaction design is that user interface and appropriate interaction model must be unobtrusive, inconspicuous and designed in such a way that imposes minimal cognitive load on the user [9], hence user interface should enable users to act and feel natural [25]. Moreover, user interface to be perceived as natural "common behaviours and mental patterns for mid-air interaction of people have to be recognized" [4]. Towards this goal, research results reveal that "bimanual interaction is perceived by the majority as more natural than unimanual" [4]. We can argue that the notion of bimanual mid-air gesture interface, with emphasis on bimanual, must be apparent in context of enabling the user to act and feel natural. However, this may be not so prominent, mainly because of the technical imitations of sensors and models applied. Nevertheless, we argue that the notion of bimanual mid-air gesture interface is a very important one. Utilizing both hands with separation of functions between dominant and non-dominant hand, Cui and Sourin have shown benefits of bimanual interaction: "mid-air interaction can be faster than 2D input device for 3D tasks" [5]. Subjective evaluation has shown that modelling using 3D bimanual mid-air interaction is easier to learn, more comfortable and "can be used with higher precision" [5].

Hindrances of bimanual mid-air interaction are related to both human factors on the one side and sensor devices and model of their usage on the other. Regarding the sensor devices, in their paper about psychophysical elements of wearability, Dunne and Smith argue that "wearability can be seen as essential both to the willingness of the user to accept and use a wearable device, and to the ability of the device to actually provide a cognitive aid" [6] thus wearability "becomes an essential part of the humancomputer interface" [6]. Innately, wearable sensor devices are not natural and as such generally impose limitations on the user making the experience of the interaction less natural by limiting the range of movements or forcing the user to apply additional muscle strain. This leads to the conclusion that "come as you are" is a paradigm that should be supported and utilized, thus acknowledging issues inherent to this approach.

Most prominent issue regarding the optical sensor such as Leap Motion regarding the bimanual mid-air interaction is limited detection range, i.e. the field of view (FOV). Research data limits the effective range of the device to the space that occupies the cube with edge length not more than $40 \mathrm{~cm}$ or even less (height being in range of $15-40 \mathrm{~cm}$ ) with resolution of $2 \mathrm{~cm} \mathrm{[5].} \mathrm{As} \mathrm{this} \mathrm{is} \mathrm{rather} \mathrm{limited} \mathrm{space}$ of operation, especially for bimanual interaction style, we are exploring a method for expanding the range of the system for bimanual mid-air interaction by using more than one sensor; this approach is similar to one described in [19].

\section{LEAP MOTION CONTROLLER}

In this paper, a Leap Motion sensor, that does not require gloves to allow interaction with the computer, will be used to detect gestures. The Leap Motion Controller is a significant step in inputting technology that could enhance interactivity with a new generation of much more useful 3D displays and may surpass the mouse as the primary input device in a three-dimensional space.

Leap Motion is a small-sized sensor capable of detecting and tracking user's hands and offers the user the opportunity to experience the digital world at a brand new level, making the overall experience more natural. It allows interaction with digital content in virtual and augmented reality by using the hands of users the same way they would use them in the real world $[11,17]$.

This mode of management opens up new interaction possibilities that can be applied in different branches and application in an interactive interface is just one of the possible examples of how to improve the learning experience in an interactive, intuitive and fun way. The device can be used in two ways. The first is when the device is on the table in a static state. Another way of using it is in virtual reality when used with stereoscopic devices like Oculus Rift. In this case, the Leap Motion device is mounted on a stereoscopic device. The user changes the view in the application by turning the head, and because of that, Leap Motion will not be in the static position but will move every time the user moves the head.

The device itself is small, $80 \mathrm{~mm}$ long, $30 \mathrm{~mm}$ wide, $11.25 \mathrm{~mm}$ tall and very light (32.4 grams), making it practical and easy to carry. The device is shown in Fig. 1 [14]. It tracks all ten fingers with a precision of one hundred millimetres and generates up to 200 frames per second. The field of view of the device is similar to the reverse pyramid whose two heights of the opposite sides close at an angle of $150^{\circ}$, where the range of reading in depth and width is up to $60 \mathrm{~cm}$ and in height from 2.5 to 60 $\mathrm{cm}$ [2]. Specifications are given by the device producer; however research shows that effective range of the device can be less than specified by producer data [5].

The $150^{\circ}$ interaction field allows us to move our hands freely in 3D space, providing the ability to move objects (virtual) and manipulate them just like in the real world.

If the hands go outside the field of vision, monitoring is lost, the sensor faces the problem of distinguishing similar gestures and position of the hands. Since the working conditions of the sensor cannot always be ideal, it can easily happen that the sensor misidentifies the exact position of the hand and fingers and the arm orientation itself. That is the reason why an error occurs in recognition.

The precision and reliability of Leap Motion was analysed by a team of engineers from the Faculty of Electrical Engineering in Ljubljana.

Two types of measurement, static and dynamic, were performed in the experiment. The static measurement was carried out. The lowest standard deviation of $0.0081 \mathrm{~mm}$ was found at $30 \mathrm{~cm}$ exactly above the device while the 
maximum of $0.49 \mathrm{~mm}$ was found in the left upper corner of the device.

The dynamic measurement was performed. Measurements have shown that the standard deviation distribution is not normal (Gaussian curve), contrary to what was expected. The biggest cause are greater deviations that occur when the observed object is moving away from the sensor, especially if the marker position would exceed the distance of $25 \mathrm{~cm}$ from the device into height. Same as in static measurement, there were instabilities in sampling rate between different measurements [7].

By analysing the obtained results, it is concluded that the device has a limited space in which the sensors give good results, where sometimes for some reason better or worse results occur due to the position of the arm in the visual field of the device [19]. The most accurate readings are obtained when the marker crosses the device because the entire object is then most visible.

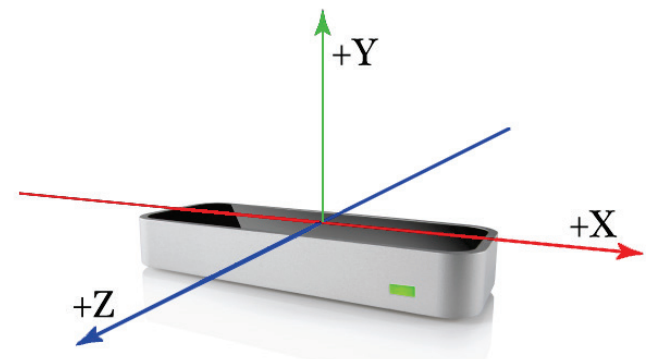

Figure 1 The right-hand gesture coordinate system of the Leap Motion Sensor. Image taken from the Leap Motion website (https://developer.leapmotion.com/documentation/v4/Leap_Axes.png)

\section{EXPERIMENTAL}

The primary goal of our experiment was to enable utilization of more natural gestures by expanding the arm movement. This goal can be achieved by increasing the size of the space in which multiple sensors are able to detect hand movement.

The precision of measurements should also be better as a repercussion of using multiple sensors, that is, at least in the space which is reachable by both sensors. However, precision of measurement is not our primary goal. Furthermore, for border areas without the reach of both sensors, precision cannot be improved. On the other hand, to successfully detect gestures, absolute precision of hand location is not significant, and will be tackled in future work.

To expand the arm movement field and reduce finger occlusion, the idea to connect two Leaps emerged. Currently, Leap Motion software does not allow two Leaps to be connected and used on the same computer at the same time. Therefore, to solve this problem and increase the interaction field, another computer will be used to collect data from another Leap and will be sent via network from one computer to the other. Two Asus G751JT laptops (i7, 16GB RAM, NVIDIA GeForce GTX970M 2GB) and DLink's 5-port switch are used to transfer data from one computer to the other.

The data obtained is transferred from one computer to the other on a real-time basis and drawn on the display of both computers. The program code for synchronization and operation of the Leap Motion sensor is made through Unity 3D software.

The data obtained is transferred from one computer to the other on a real-time basis and drawn on the display of both computers. The program code for synchronization and operation of the Leap Motion sensor is made through Unity 3D software.

The method that decides from which data a hand will be drawn is based on a coordinate system in which Leap Motion works. Coordinate hands are looked by an identifier, in our case, the centre of the palm is taken, a propos centre of the object. The following picture shows the location of the palm.

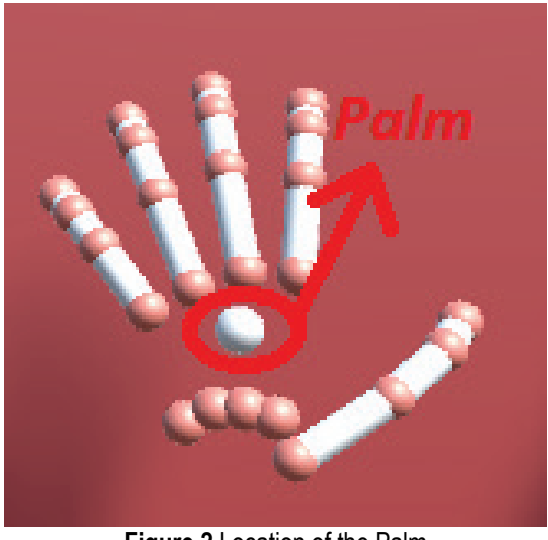

Figure 2 Location of the Palm

The method works on the principle that computers communicate with each other and exchange palm coordinates. Using these coordinates, it determines which Leap Motion is closer to the Palm. Drawing the hands is based on the coordinate system $x, y, z$.

Since it wants to expand the area interactions by $x$ coordinates, it compares the $x$ coordinates of both Leaps. For example, if the palm point of the received $x$ coordinate closer to the left Leap is then drawn from the left Leap, which implies that the $x$ coordinate is away from right Leap, although both Leaps see the palm.

Current measurements have shown that the system works well at a distance of $20 \mathrm{~cm}, 40 \mathrm{~cm}, 60 \mathrm{~cm}$ and 70 $\mathrm{cm}$. Moreover, each Leap Motion sensor can recognize the point of the palm at its far left or right side up to about 30 $\mathrm{cm}$. We come to the information that, by this type of connection, i.e. networking, the user interaction field has expanded to $130 \mathrm{~cm}$, which is more than enough for each interaction in the virtual world, and that the user has a more natural user interface.

In the operation of two Leaps, there is no noticeable latency in recognizing and drawing of hands on both laptops.

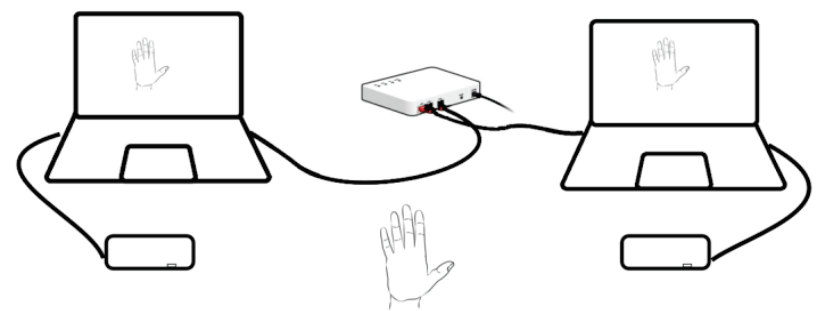

Figure 3 Connecting two Leap Motion sensors Fig. 4 depicts out testing setup. 


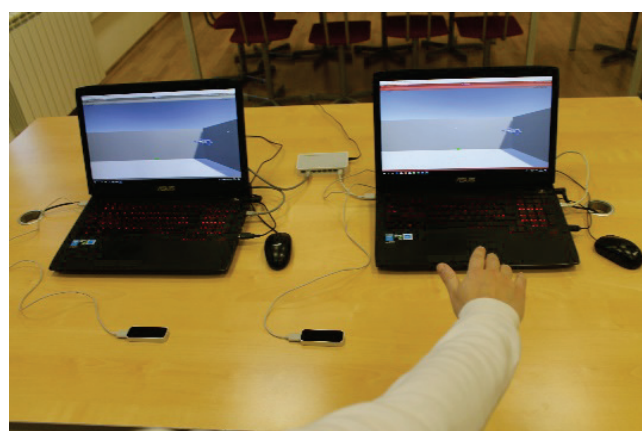

Figure 4 Hand on the right in FOV of right sensor

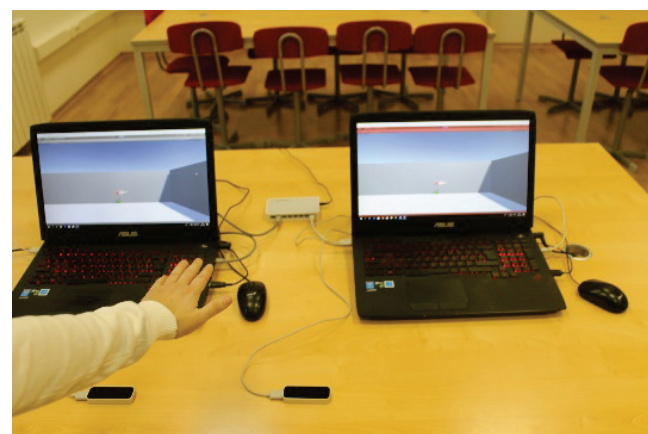

Figure 5 Hand in the middle tracked by both left and right sensor

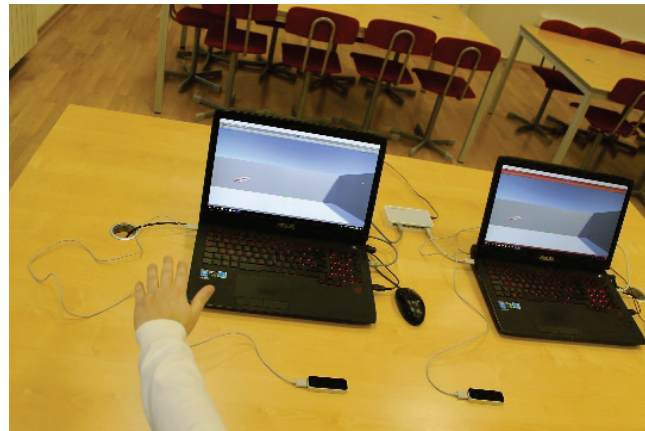

Figure 6 Hand on the left in FOV of the left sensor

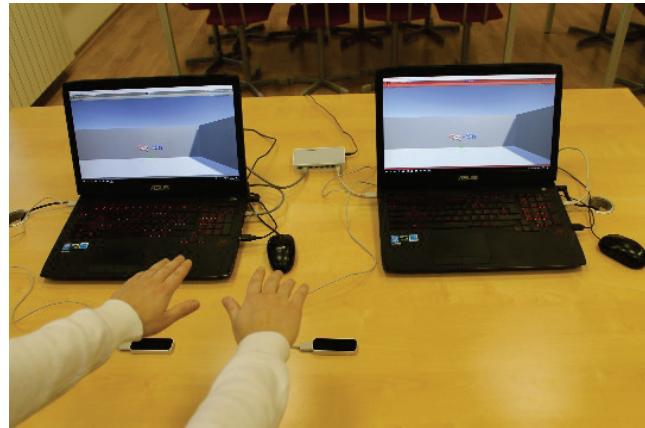

Figure 7 Two hands in the middle in FOV of both sensors - software depicts data from both local and remote sensor

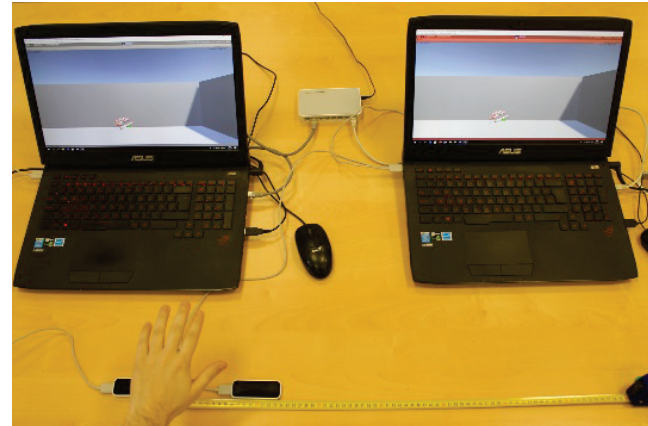

Figure $8 \mathrm{Hand}$ in the middle of the FOV of both sensors - at $20 \mathrm{~cm}$

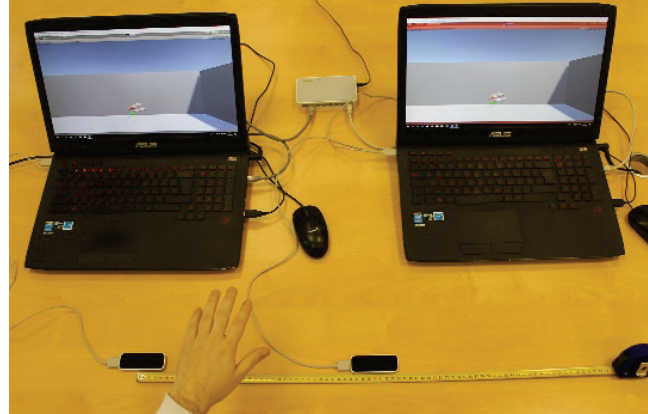

Figure 9 Hand in the middle of the FOV of both sensors - at $40 \mathrm{~cm}$

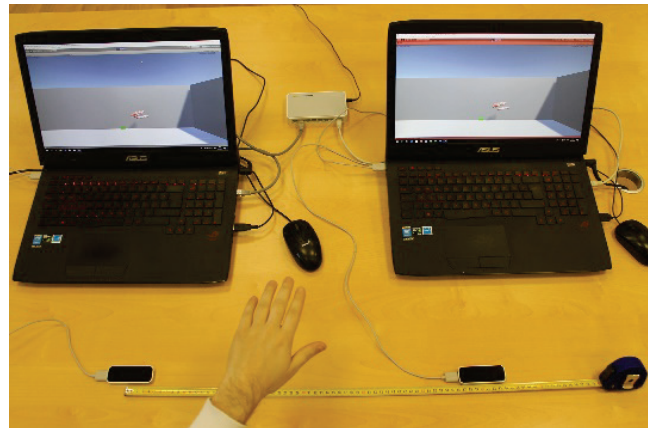

Figure $10 \mathrm{Hand}$ in the middle of the FOV of both sensors - at $60 \mathrm{~cm}$

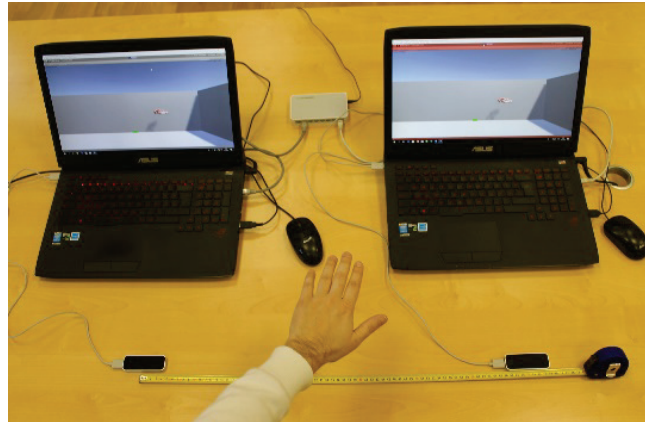

Figure 11 Hand in the middle of the FOV of both sensors - at $70 \mathrm{~cm}$

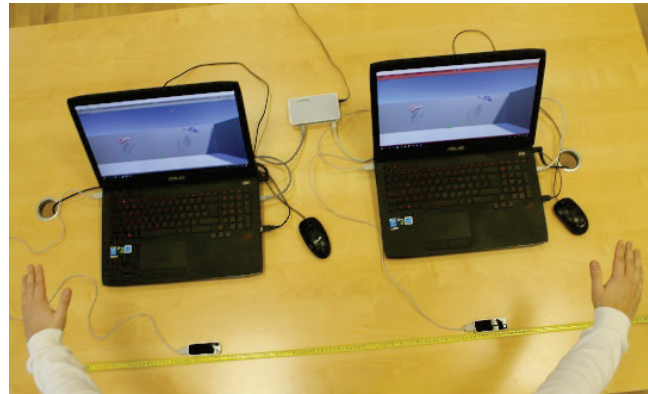

Figure 12 Hand outside of the FOV of both sensors - at $70 \mathrm{~cm}$

Moving one hand across both sensors is shown in Figs. 4, 5 and 6. Both computers use the same program code. Each computer reads data from a local sensor (connected to a computer), displays read data on the screen, and displays hands painted in red and blue. Fig. 1 shows the hand that enters the right side of the right-hand field of the right sensor; in this position the arm is too far to detect the left sensor. If the user moves his arm further to the left, as shown in Fig. 5, the hand is placed in the FOV of both sensors. In this position, both sensors detect a hand and a computer whose sensor closer to the palm points to the hand. Fig. 6 shows moving the left-hand and arm to the left, above the left sensor, and outside the FOV of the right 
sensor. The computer screens, similar to Fig. 4, point to the left-hand side, as they display data from the nearest sensor.

Fig. 7 shows a two-handed interaction between two Leap Motion sensors, where both sensors recognize left and right-hand and each extractor of recognized hands, depending on which sensor is closer to the Palm point.

Figs. 8, 9, 10, 11 and 12 show the distance from each sensor. It is shown that the sensors can work at a distance of $70 \mathrm{~cm}$, which increases the area of interaction by $x$ coordinates.

\section{CONCLUSION}

This paper gives an overview of Leap Motion sensors. Leap Motion is a device that monitors the user's hand movement and is a step forward in the human-computer interaction. The device is small and easy to carry and has impressive specifications. Occlusion is the main problem when using one sensor and the two-sensor data fusion will try to find the optimal distance and the angle at which the occlusion will be the smallest.

The connection of two Leap Motion devices is successfully synchronized by connecting two laptops. This research has improved interactions and increased the range of hand movements within the application. Increasing the range of motion gives a better impression of a Natural user interface. It is suitable for application where we rely heavily on the hand input mode.

Leap is a revolutionary motion-tracking product that, with the addition of two sensors, can be used very well in applications. This will bring the whole experience of the human-computer interaction to a new level.

\section{Acknowledgements}

This paper describes the results of research being carried out within the project "Centar održivog razvoja" ("Center of sustainable development"), co-financed by the European regional development fund and implemented within Operational Programme Competitiveness and Cohesion 2014 - 2020, based on the call "Investing in Organizational Reform and Infrastructure in the Research, Development and Innovation Sector".

\section{REFERENCES}

[1] Bui, T. D. \& Nguyen, L. T. (2007). Recognizing Postures in Vietnamese Sign Language with MEMS Accelerometers. IEEE Sensors Journal, 7(5), 707-712. https://doi.org/10.1109/JSEN.2007.894132

[2] Cogan, A. (2017). How Does the Leap Motion Controller Work? Retrieved June 5, 2017, from http://blog.leapmotion.com/hardware-to-software-howdoes-the leap-motion-controller-work/

[3] Cui J., Fellner D. W., Kuijper A., \& Sourin A. (2016) MidAir Gestures for Virtual Modeling with Leap Motion. In: Streitz N., Markopoulos P. (eds) Distributed, Ambient and Pervasive Interactions. DAPI 2016. Lecture Notes in Computer Science, vol. 9749. Springer, Cham. https://doi.org/10.1007/978-3-319-39862-4_21

[4] Cui, J., Kuijper, A., Fellner, D. W., \& Sourin, A. (2016). Understanding People's Mental Models of Mid-Air Interaction for Virtual Assembly and Shape Modeling. Proceedings of the $29^{\text {th }}$ International Conference on
Computer Animation and Social Agents - CASA '16, 139146. https://doi.org/10.1145/2915926.2919330

[5] Cui, J. \& Sourin, A. (2017). Interactive shape modeling using leap motion controller. SIGGRAPH Asia 2017 Technical Briefs, SA 2017. https://doi.org/10.1145/3145749.3149437

[6] Dunne, L. E. \& Smyth, B. (2007). Psychophysical elements of wearability. Proceedings of the SIGCHI Conference on Human Factors in Computing Systems - CHI '07, 299. https://doi.org/10.1145/1240624.1240674

[7] Guna, J., Jakus, G., Pogačnik, M., Tomažič, S., \& Sodnik, J. (2014). An Analysis of the Precision and Reliability of the Leap Motion Sensor and Its Suitability for Static and Dynamic Tracking. Sensors, 14(2), 3702-3720. https://doi.org/10.3390/s140203702

[8] Hincapié-Ramos, J. D., Guo, X., Moghadasian, P., \& Irani, P. (2014). Consumed Endurance: A metric to quantify arm fatigue of mid-air interactions. Proceedings of the $32^{\text {nd }}$ Annual ACM Conference on Human Factors in Computing Systems - CHI '14, 1063-1072. https://doi.org/10.1145/2556288.2557130

[9] Johnson, J. (2010). Designing with the mind in mind: simple guide to understanding user interface design rules. Children $\left(1^{\text {st }}\right.$ ed). Morgan Kaufmann Publishers Inc. Retrieved from http://books.google.com/books?hl=

en\&amp;lr=\&amp;id=woVADVwtL1gC\&amp;oi=fnd\&am p;pg $=$ PP2 \&amp; dq $=$ Designing + with + the + mind + in + mind $\&$ amp;ots=vo7eeBASon\&amp;sig=V2ov3lai0ZQ1RaR9zM93 $\mathrm{FbGu} \mathrm{Rw}$

[10] Jude, A., Poor, G. M., \& Guinness, D. (2014). Personal space: user defined gesture space for GUI interaction. Proceedings of the Extended Abstracts of the $32^{\text {nd }}$ Annual ACM Conference on Human Factors in Computing Systems - CHI EA '14, 1615-1620. https://doi.org/10.1145/2559206.2581242

[11] Koo, B., Kim, J., \& Cho, J. (2014). Leap Motion Gesture Based Interface for Learning Environment by Using Leap Motion. In Proceedings of HCI Korea (pp. 209-214). South Korea: Hanbit Media, Inc. Retrieved from http://dl.acm.org/citation.cfm?id=2729485.2729516

[12] Koutsabasis, P. \& Domouzis, C. K. (2016). Mid-Air Browsing and Selection in Image Collections. Proceedings of the International Working Conference on Advanced Visual Interfaces - AVI '16, 21-27. https://doi.org/10.1145/2909132.2909248

[13] Krupka, E., Karmon, K., Bloom, N., Freedman, D., Gurvich, I., Hurvitz, A., ... Bar-Hillel, A. (2017). Toward Realistic Hands Gesture Interface: Keeping It Simple for Developers and Machines. In Proceedings of the 2017 CHI Conference on Human Factors in Computing Systems (pp. 1887-1898). New York, NY, USA: ACM. https://doi.org/10.1145/3025453.3025508

[14] Leap Motion, Inc, Leap Motion Controller. (n.d.). Retrieved June 3, 2017, from https://www.leapmotion.com/product

[15] McCartney, R., Juan, J., \& Bischof, H.-P. (2015). Gesture Recognition with the Leap Motion Controller. In Proc. of the IPCV'15 - The $19^{\text {th }}$ International Conference on Image Processing, Computer Vision, \& Pattern Recognition. Retrieved from https://scholarworks.rit.edu/other/857/

[16] Monnai, Y., Hasegawa, K., Fujiwara, M., Yoshino, K., Inoue, S., \& Shinoda, H. (2014). HaptoMime: Mid-Air Haptic Interaction with a Floating Virtual Screen. Proceedings of the $27^{\text {th }}$ Annual ACM Symposium on User Interface Software and Technology - UIST '14, 663-667. https://doi.org/10.1145/2642918.2647407

[17] Nandy, A. (2016). Leap Motion for Developers ( $1^{\text {st }}$ ed.). Berkely, CA, USA: Apress. https://doi.org/10.1007/978-1-4842-2550-9 
[18] Oviatt, S. (2003). User-centered modeling and evaluation of multimodal interfaces. Proceedings of the IEEE, 91(9), 1457-1468. https://doi.org/10.1109/JPROC.2003.817127

[19] Placidi, G., Cinque, L., Polsinelli, M., \& Spezialetti, M. (2018). Measurements by a LEAP-Based Virtual Glove for the Hand Rehabilitation. Sensors, 18(3). https://doi.org/10.3390/s18030834

[20] Shrivastava, R. (2013). A hidden Markov model based dynamic hand gesture recognition system using OpenCV. In $3^{\text {rd }}$ IEEE International Advance Computing Conference (IACC), 947-950. https://doi.org/10.1109//AdCC.2013.6514354

[21] Tarchanidis, K. N. \& Lygouras, J. N. (2003). Data glove with a force sensor. IEEE Transactions on Instrumentation and Measurement, 52(3), 984-989. https://doi.org/10.1109/TIM.2003.809484

[22] Triesch, J. \& von der Malsburg, C. (1998). Robotic Gesture Recognition by Cue Combination. In J. Dassow \& R. Kruse (Eds.), Informatik '98, Berlin, Heidelberg: Springer Berlin Heidelberg, 223-232. https://doi.org/10.1007/978-3-642-72283-7_22

[23] Turk, M. (2014). Review Article: Multimodal Interaction: A Review. Pattern Recogn. Lett., 36, 189-195. https://doi.org/10.1016/j.patrec.2013.07.003

[24] Wachs, J. P., Kölsch, M., Stern, H., \& Edan, Y. (2011). Vision-based hand-gesture applications. Communications of the ACM, 54(2), 60. https://doi.org/10.1145/1897816.1897838

[25] Wigdor, D. \& Wixon, D. (2011). Brave NUI World: Designing Natural User Interfaces for Touch and Gesture ( $1^{\text {st }}$ ed.). Morgan Kaufmann Publishers Inc. https://doi.org/10.1016/B978-0-12-382231-4.X0001-9

\section{Contact information:}

Nenad BRESLAUER, Senior Lecturer

Polytechnic of Međimurje in Čakovec,

Bana Josipa Jelačića 22a

40000 Čakovec, Croatia

E-mail: nbreslauer1@mev.hr

Irena GALIĆ, PhD, Associate Professor

J. J. Strossmayer University of Osijek

Faculty of Electrical Engineering, Computer Science and Information

Technology Osijek

Kneza Trpimira 2B,

31000 Osijek, Croatia

E-mail: irena@etfos.hr

Mihale KUKEC, PhD, Assistant Professor

Polytechnic of Međimurje in Čakovec

Bana Josipa Jelačića 22a

40000 Čakovec, Croatia

E-mail: mkukec@mev.hr

Ivan SAMARDŽIĆ, PhD, Professor with Tenure

J. J. Strossmayer University of Osijek,

Mechanical Engineering Faculty in Slavonski Brod,

Trg Ivane Brlić-Mažuranić 2,

35000 Slavonski Brod, Croatia

E-mail: isamar@sfsb.hr 